\title{
A Robust Cooperative Spectrum Sensing-Assisted Multiuser Resource Allocation Scheme
}

\author{
Jun Du, ${ }^{1}$ Daoxing Guo, ${ }^{1}$ Bangning Zhang, ${ }^{1}$ and Yunxia Su${ }^{2}$ \\ ${ }^{1}$ College of Communications Engineering, PLA University of Science \& Technology, Nanjing, Jiangsu 210007, China \\ ${ }^{2}$ Unit 72241, PLA, Jinan, Shandong 250029, China
}

Correspondence should be addressed to Jun Du; dujun607@gmail.com

Received 27 April 2014; Accepted 25 July 2014

Academic Editor: Guido Maione

Copyright (C) 2015 Jun Du et al. This is an open access article distributed under the Creative Commons Attribution License, which permits unrestricted use, distribution, and reproduction in any medium, provided the original work is properly cited.

Cognitive radio (CR), which is proposed as a solution for spectrum scarcity, imposes some threats to the network. One severe attack to cognitive radio network is the primary user emulation attack (PUEA), in which an attacker may transmit its signal with high power or mimic specific features of the primary user's signal to prevent secondary users from accessing the licensed spectrum. In this paper, we study a subcarrier and power allocation problem for orthogonal frequency division multiple access-(OFDMA-) based CR systems in the presence of PUEA. To maximize the system throughput while keeping the interference introduced to the primary user (PU) below given thresholds with a certain probability, a joint design of a robust cooperative spectrum sensing and a resource allocation scheme is proposed. In the proposed scheme, the inaccurate classification of PU signals and PUEA signals provided by robust cooperative spectrum sensing is utilized by resource scheduling module. To further exploit the underutilized spectrum bands, we also evaluate the performance of the proposed scheme in the hybrid overlay/underlay spectrum access mechanism. Numerical results demonstrate the effectiveness of the proposed scheme compared to conventional scheme regardless of the number of SUs or the kind of spectrum access mechanism being used.

\section{Introduction}

Cognitive radio networks (CRNs) are expected to bring a revolution to the spectrum scarcity problem through intelligent use of the underutilized or the free spectrum bands [1]. In CRNs, a secondary (unlicensed) user (SU) can access the licensed bands under the condition that the induced interference to the primary (licensed) users (PUs) does not reach an unacceptable level. In general, to exploit unused and underutilized spectrum bands, an SU has three spectrum sharing approaches for a dynamic spectrum access mechanism [2], namely, the underlay spectrum access mechanism (USAM), the overlay spectrum access mechanism (OSAM), and hybrid overlay/underlay spectrum access mechanism (HOUSAM) [3]. According to the OSAM, SUs access the spectral bands only when they are not being used by the PUs; in the USAM, the PUs and SUs can coexist in the same spectral band which means the SUs can utilize the underutilized frequency bands of the PUs. In HOUSAM, the SU can transmit in both the unused and underutilized spectrum bands. It improves the throughput of the secondary network while maintaining a harmless interference to the PU.

Orthogonal frequency division multiplexing (OFDM) is a promising candidate for CRNs [4]. With OFDM, the SU has the ability to flexibly fill the spectral gaps left by PUs and/or utilize the underutilized frequency bands of the PUs, by disabling or decreasing the power allocated to a set of subcarriers. In a conventional OFDM system, to maximize the efficiency of the spectral resources utilization, a powerallocation algorithm is proposed in [5]. In an orthogonalfrequency-division multiple-access- (OFDMA-) based system, to exploit the time-varying nature of fading gains across users in a given subcarrier, a subcarrier allocation algorithm is proposed in [6]. For conventional OFDMA wireless systems, resource allocation algorithms have been studied extensively [7]. Using these resource allocation algorithms directly into CRNs may result in higher mutual interference in the PUs' band, since they coexist in side-by-side bands and the nonorthogonality of transmitting signals. Recently, 
resource allocations in OFDM-based CRNs have received a great deal of attention.

Finding unused and underutilized spectrum bands in CRNs is based on the information gathered from SUs' spectrum sensing reports. However, in the hostile network environment, an attacker can mount primary user emulation attack (PUEA) to affect SUs' reports. In such scenario, an attacker transmits signals whose characteristics resemble that of the PU transmitter, misleading the SUs to believe that the spectrum bands are occupied by the PU. Robust spectrum sensing [8] and resource allocation are two key enabling technologies for cognitive radio (CR). The former mainly concerns how to reliably recognize the existence of the unused spectrum bands and the latter concerns how to maximize the utilization of the recognized unused spectrum bands. For instance, in [9] a hybrid PUEA defense strategy based on a combination of energy detection and variance detection is proposed. The authors in [10] proposed a robust cooperative spectrum sensing that considers an always present PUEA. For a smart PUEA, who is able to perform spectrum sensing and send its signal in a planned way, the authors in [11] proposed a new spectrum sensing scheme. In [12], the authors study resource allocation problem for OFDMA-based cognitive radio systems. A subcarrier and power allocation schemes for a hybrid overlay and underlay spectrum access mechanism are proposed in [13]. In [14], a distributed power allocation algorithm for underlay CRNs is proposed, where channel gains between SUs and PU base stations are uncertain.

In most of existing designs for CRNs, robust spectrum sensing and resource allocation are implemented separately. That is, in the first step, robust spectrum sensing makes a relatively reliable decision on whether the primary signal is present or not. Then this decision is transferred to the next step. Based on the information got from the first step, a resource scheduling module could accomplish the power allocation and/or spectrum band assignment task. In this paper, we study the joint design of robust spectrum sensing and resource allocation in OFDMA-based cognitive radio systems. The feasibility of this idea is as follows.

Firstly, robust spectrum sensing has the ability to make a relatively reliable classification of PU signals and PUEA signals not just distinguish vacant bands from occupied bands. Secondly, conventional resource allocation assumes a secure environment and did not utilize the information of imperfect classification of PU signals and PUEA signals got from robust spectrum sensing. Recently, a valuable work [15] has revealed that robust resource allocation, which takes into account possible spectrum sensing errors occurring due to the presence of PUEA signals in the environment, is more efficient than conventional resource allocation. The scenario that the authors in [15] considered is composed of a primary and a secondary transceiver. This scenario is simple and the proposed resource allocation scheme is based on OSAM, which does not utilize the underused spectral regions.

Due to the uncertainty present in the secondary base station- (SBS-) to-SU channel, underlay SU subcarriers may experience relatively better channel qualities, whereas the channel quality between the SBS and an overlay SU subcarrier

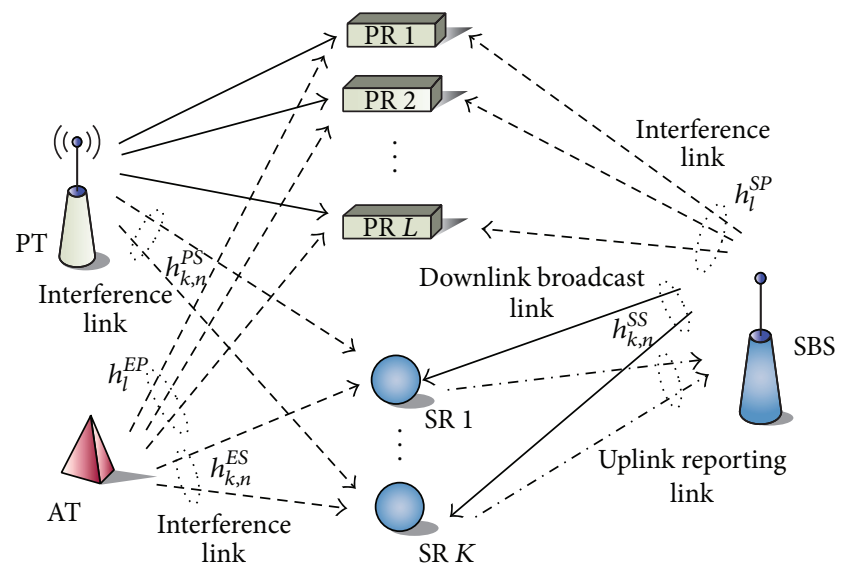

PT: primary users' transmitter SR: secondary user's receiver PR: primary user's receiver AT: attacker's transmitter SBS: secondary base station

FIgURE 1: System model.

can be poor. Therefore, to increase the overall spectrum utilization, the SUs may need to utilize not only the unused frequency bands but also underutilized frequency bands in a given geographical location and in a particular time. In this paper, considering the factor of the not fully reliable spectrum sensing results due to the PUEA, we propose a robust cooperative spectrum sensing-assisted multiuser resource allocation scheme (Rope-Alone) for OFDM-based CRNs. We showed that joint design of a robust cooperative spectrum sensing and a resource allocation outperforms the separated design. The proposed Rope-Alone can be used not only with an OSAM but also with a HOUSAM.

The rest of the paper is organized as follows. In Section 2, the system model is introduced and the problem discussed in this paper is formulated as an optimization problem. Section 3 presents the details of the solution for our proposed Rope-Alone. The results of numerical tests are presented in Section 4, followed by conclusions in Section 5 .

\section{System Model}

2.1. Overall Description. In this paper, as shown in Figure 1, we consider a downlink transmission scenario consisting of a centralized primary user network $(\mathrm{PN})$ and a centralized cognitive radio network. An attacker attempts to fool the SUs in the CRN by transmitting PUEA signals, whereas the CRN endeavors to distinguish PU signals from PUEA signals and arrive at an optimal resource allocation scheme. As shown in Figure 2, the total available bandwidth licensed to the PU is equal to $W$ which is divided into $N$ subcarriers of equal width $\Delta f . \mathcal{N}=\{1, \ldots, N\}$ is the set of subcarriers. The CRN which employs an OFDM scheme includes a secondary base station (SBS) and $K$ SUs and operates on a frameby-frame basis. $\mathscr{K}=\{1, \ldots, K\}$ is the set of SUs. At the beginning of each frame, each $\mathrm{SU}_{i} i \in \mathcal{N}$ carries out robust spectrum sensing to determine the vacant subcarriers and distinguish PU signals from PUEA signals in the occupied 


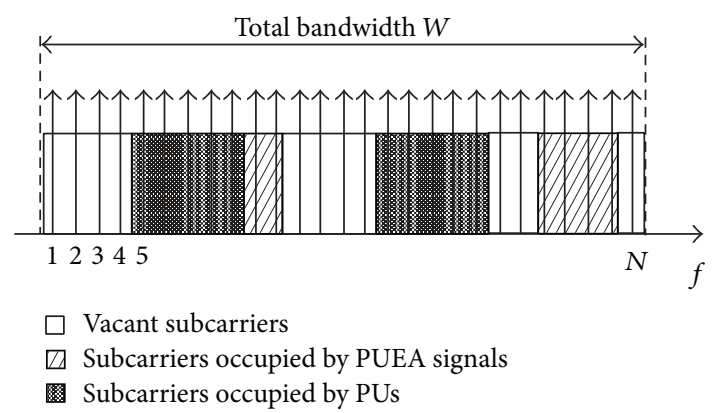

FIgURE 2: Example of the total available bandwidth.

subcarriers. Then, each $\mathrm{SU}_{i}$ sends its sensing results through the uplink reporting link to the SBS. Based on the reports collected from the SUs, we assume the SBS can perfectly differentiate vacant subcarriers from occupied subcarriers. Also, we assume that the SBS can distinguish between the PU and the PUEA signals in occupied subcarriers imperfectly. With the imperfect knowledge of the PU activity in occupied subcarriers, the SBS performs resource allocation, that is, channel or spectrum band assignment and power allocation, and feeds back the decisions to all SUs through the downlink broadcast link.

In this paper, perfect synchronization is assumed between FC and SU. This is because the IEEE 802.22 standard has defined Quiet Periods (QPs) where spectrum sensing is performed and SUs are not allowed to transmit. CRNs can synchronize their QPs through an offset synchronization technique using beacons [16]. In a centralized CRN, the FC distributes synchronization information through the control channel [17]. It is reasonable to assume that SUs are able to synchronize with the FC.

In downlink transmission scenario shown in Figure 1, there are four interference links: (1) between the primary user's transmitter (PT) and the secondary user's receiver (SR), let $h_{k, n}^{P S}$ denote the channel fading gain between the PT and the $k$ th SR in the $n$th subcarrier; (2) between the attacker's transmitter (AT) and the primary user's receiver (PR), let $h_{l}^{E P}$ denote the channel fading gain between the AT and the PR in the lth subcarrier; (3) between the AT and the SR, let $h_{k, n}^{E S}$ denote the channel fading gain between the AT and the $k$ th SR in the $n$th subcarrier; (4) between the SBS and the PR, let $h_{l}^{S P}$ denote the channel fading gain between the SBS and the PR in the $l$ th subcarrier. Furthermore, the link between the SBS and SR is the downlink broadcast link; let $h_{k, n}^{S S}$ denote the channel fading gain between the SBS and the $k$ th SR in the $n$th subcarrier. These instantaneous channel fading gains are assumed to follow a Rayleigh distribution and we assume these instantaneous fading gains are known at the SBS. Specifically, we assume that the SU can estimate instantaneous fading gains and report to the SBS through the uplink reporting link [18].

2.2. Modeling of the Imperfect Robust Cooperative Spectrum Sensing. Robust spectrum sensing can not only distinguish vacant subcarriers from occupied subcarriers, but also make a relatively reliable decision on the classification of PU signals and PUEA signals. In order to exploit this extra information, we will quantify the accuracy of classification and provide this to the subsequent resource allocation module to improve the spectrum utilization efficiency.

Each SU senses the subcarriers periodically and makes a local decision about the presence of the PU signals and PUEA signals based on its own observations. The local decisions are to be sent to the SBS in consecutive time slots based on a time division multiple access (TDMA) scheme through the uplink reporting link. The reported local decisions are combined at the SBS, as shown in Figure 2; all of these subcarriers $S=\{1, \ldots, N\}$ will be divided into three parts [15]: the set of vacant subcarriers $V$, the set of subcarriers $O^{P}$ occupied by the PU, and the set of subcarriers $O^{A}$ occupied by the PUEA. Note that intersymbol interference (ISI) and intercarrier interference (ICI) are two factors of degrading the OFDM system performance. The shorter time of SU occupancy of the estimated free carriers may result in ISI.

Furthermore, mobility of the SUs generates Doppler frequency spread, which may destroy the orthogonality between subcarriers. The loss of orthogonality (of the carriers) at the SUs results in ICI [19]. Many methods have been proposed to combat ICI. Among these methods, adaptive subcarrier bandwidth is one of the representatives. The authors in [20] reveal that increasing the subcarrier bandwidth (i.e., decreasing $N$ ) can reduce ICI. However, as the subcarrier bandwidth increases, the ISI also increases. Therefore, the subcarrier bandwidth selection is a tradeoff between decreasing the subcarrier bandwidth to eliminate the ISI and increasing the subcarrier bandwidth to reduce the ICI.

We can write

$$
S=V \cup O^{P} \cup O^{A}, \quad O^{P} \cap O^{A}=\varnothing .
$$

Due to the imperfect robust spectrum sensing, there would be errors in classification of $O^{P}$ and $O^{A}$. Let $p_{l}$ denote the real state in which the PU is sending signals over subcarrier $l\left(l \in O^{P} \cup O^{A}\right)$, and let $\widehat{p}_{l}$ denote the estimate of $p_{l}$ by robust spectrum sensing. Similarly, we denote $e_{l}$ to indicate the real state in which the attacker is sending PUEA signals over subcarrier $l\left(l \in O^{P} \cup O^{A}\right)$, and let $\widehat{e}_{l}$ denote the estimate of $e_{l}$ by robust spectrum sensing.

So when the signal is from the $\mathrm{PU}$, the probability that the SBS falsely classifies it as from the attacker is $p_{f}^{E}=\operatorname{Pr}\left(\widehat{e}_{l} \mid p_{l}\right)$. When the signal is from the attacker, the probability that the SBS falsely classifies it as from the $\mathrm{PU}$ is $p_{f}^{P}=\operatorname{Pr}\left(\widehat{p}_{l} \mid e_{l}\right)$. Note that the probability of detection of PU signals and PUEA signals can be written as $p_{d}^{P}=\operatorname{Pr}\left(\hat{p}_{l} \mid p_{l}\right)=1-p_{f}^{E}$ and $p_{d}^{E}=\operatorname{Pr}\left(\widehat{e}_{l} \mid e_{l}\right)=1-p_{f}^{P}$, respectively.

Here, let us define $\operatorname{Pr}\left(p_{l} \mid \widehat{p}_{l}\right)$ as the probability that the SBS classifies the signals to be from the PU when it is indeed from the PU, and $\operatorname{Pr}\left(e_{l} \mid \widehat{e}_{l}\right)$ as the probability that the SBS classifies the signals to be from the PUEA when it is indeed from the PUEA. Hence, we use $\alpha_{l}^{P}$ and $\alpha_{l}^{A}$ to evaluate the accuracy of classification for the PU signals and the PUEA 
signals. Formally, these two probabilities which will be used in the subsequent resource allocation are defined as

$$
\begin{aligned}
& \alpha_{l}^{P}=\operatorname{Pr}\left(p_{l} \mid \widehat{p}_{l}\right)=\frac{p_{d}^{P} \operatorname{Pr}\left(p_{l}\right)}{\operatorname{Pr}\left(\widehat{p}_{l}\right)}, \\
& \alpha_{l}^{A}=\operatorname{Pr}\left(e_{l} \mid \widehat{e}_{l}\right)=\frac{p_{d}^{E} \operatorname{Pr}\left(e_{l}\right)}{\operatorname{Pr}\left(\widehat{e}_{l}\right)},
\end{aligned}
$$

respectively. The probability of $\operatorname{Pr}\left(\widehat{p}_{l}\right)$ in (2) and the probability of $\operatorname{Pr}\left(\widehat{e}_{l}\right)$ in (3) can be rewritten as $\operatorname{Pr}\left(\widehat{p}_{l}\right)=p_{d}^{P} \operatorname{Pr}\left(p_{l}\right)+$ $p_{f}^{P} \operatorname{Pr}\left(e_{l}\right)$ and $\operatorname{Pr}\left(\widehat{e}_{l}\right)=p_{d}^{E} \operatorname{Pr}\left(e_{l}\right)+p_{f}^{E} \operatorname{Pr}\left(p_{l}\right)$, respectively, where $\operatorname{Pr}\left(p_{l}\right)$ denotes the probability of presence of the PU which is signal in the subcarrier $l, \operatorname{Pr}\left(e_{l}\right)$ denotes the probabilities of presence of the PUEA signal in the subcarrier $l$.

2.3. Modeling of the Resource Allocation Problem in the Presence of an Attacker. In this paper, the goal of resource allocation is to maximize the total transmission rate of $K$ SUs while keeping total interference introduced to the PU subcarriers below a certain threshold and total power below a constraint.

The capacity for the $n$th subcarriers used by the $k$ th SU is calculated via the Shannon formula as [21]

$$
r_{k, n}=\Delta f \log _{2}\left(1+\frac{p_{k, n}\left|h_{k, n}^{S S}\right|^{2}}{\sigma^{2}+i_{k, n}+j_{k, n}}\right)
$$

where $p_{k, n}$ is the transmission power of the $k$ th SU for the $n$th subcarrier, $\sigma^{2}$ denotes the additive white Gaussian noise (AWGN) variance, $i_{k, n}$ denotes the interference introduced to the $n$th subcarriers of the $k$ th SU due to the transmission of all PUs, and $j_{k, n}$ denotes the interference introduced to the $n$th subcarriers of the $k$ th SU due to the transmission of PUEA signals.

The sum capacity of the $k$ th SU is

$$
r_{k, n}=\Delta f \sum_{n=1}^{N} C_{k, n} \log _{2}\left(1+\frac{p_{k, n}\left|h_{k, n}^{S S}\right|^{2}}{\sigma^{2}+i_{k, n}+j_{k, n}}\right)
$$

where $C_{k, n}$ can have only value of either 1 or 0 , indicating whether the $n$th subcarrier is used by the $k$ th SU or not, as only one SU can occupy a particular subcarrier.

Mathematically, the objective is to find the optimal exclusive subcarrier assignment policy $C_{k, n}$ and power allocation policy $p_{k, n}$ jointly such that the CRN system throughput is maximized, while satisfying the interference temperature constraint required by the PU on each channel and the power budget of the SBS simultaneously; that is,

$$
\begin{array}{ll}
\max _{C_{k, n}, p_{k, n}} & \Delta f \sum_{k=1}^{K} \sum_{n=1}^{N} C_{k, n} \log _{2}\left(1+\frac{p_{k, n}\left|h_{k, n}^{S S}\right|^{2}}{\sigma^{2}+i_{k, n}+j_{k, n}}\right) \\
\text { s.t. } \quad \mathrm{C} 1: \operatorname{Pr}\left(I(l) \leq I_{\mathrm{th}}^{(l)}\right) \geq a, \quad l \in O^{P} \cup O^{A} \\
\mathrm{C} 2: C_{k, n}=\{0,1\}, \quad \forall k \in \mathscr{K}, \quad \forall n \in \mathscr{N} \\
\mathrm{C} 3: \sum_{k=1}^{K} C_{k, n}=1, \quad \forall n \in \mathscr{N} \\
\mathrm{C} 4: p_{k, n} \geq 0, \quad \forall k \in \mathscr{K}, \quad \forall n \in \mathscr{N} \\
\mathrm{C} 5: \sum_{k=1}^{K} \sum_{n=1}^{N} C_{k, n} p_{k, n} \leq P_{T},
\end{array}
$$

where $P_{T}$ denotes the total power budget, $I(l)$ denotes the interference introduced to the $l$ th subcarriers of the PU due to the transmission of all SUs, and $I_{\text {th }}^{(l)}$ denotes the interference threshold for the $l$ th PU subcarriers. $\mathrm{Cl}$ are the interference constraints for all PUs subcarriers; it can be guaranteed that the interference introduced to the $l$ th PU subcarriers remains below a specified interference threshold $I_{\text {th }}^{(l)}$ with specified probability $a$ [12]. C2 and C3 indicate each subcarrier can be allocated to only one SU. C4 and C5 are the transmitter power constraints.

In the subsequent section, this optimization problem will be deduced in detail; an optimal subcarrier-and-power allocation can be obtained.

\section{Robust Cooperative Spectrum Sensing- Assisted Multiuser Resource Allocation}

The optimization problem considered here is similar to the conventional resource allocation problem, except for the added interference to the CRN due to the presence of the attacker and the modified interference constraints $\mathrm{Cl}$ got from robust cooperative spectrum sensing algorithm. In the following, we derive three kinds of interferences. Then, we formulate the objective function with the constraints in (6) and propose the Rope-Alone.

3.1. PU-SU Interference. This is the PN interference on the SU subcarrier. Since the waveform of the PU activity is unknown, an elliptically filtered white noise process is assumed as PU signal [22]. We assume that $\Phi_{l}^{\mathrm{PU}}(\omega)$ is the power spectrum density of the PU signal at subcarrier $l$; then the interference introduced by the $l$ th PU subcarrier to the SU $k$ that is using subcarrier $n$ can be calculated as follows [22]:

$$
i_{k, n}^{\mathrm{PU}}(l)=\left|h_{k, n}^{P S}\right|^{2} \int_{d_{n, l}-\Delta f / 2}^{d_{n, l}+\Delta f / 2} \Phi_{l}^{\mathrm{PU}}(\omega) d \omega,
$$

where $d_{n, l}$ is the frequency distance between subcarrier $n$ and subcarrier $l$. 
The interference introduced by the PU subcarriers that are correctly detected in the set $O^{P}$, imposed to the SU $k$ that is using subcarrier $n$, can be written as

$$
i_{k, n}^{P}=\sum_{l \in O^{P}} \alpha_{l}^{P} i_{k, n}^{\mathrm{PU}}(l)
$$

The interference introduced by the PU subcarriers that are incorrectly detected as PUEA signals in the set $O^{A}$, imposed to the SU $k$ that is using subcarrier $n$, can be written as

$$
i_{k, n}^{A}=\sum_{l \in O^{A}}\left(1-\alpha_{l}^{A}\right) i_{k, n}^{\mathrm{PU}}(l)
$$

Thus, the interference introduced by the PU signal to the $\mathrm{SU} k$ that is using subcarrier $n$ can be written as

$$
i_{k, n}=i_{k, n}^{P}+i_{k, n}^{A}
$$

3.2. PUEA-SU Interference. This is the PUEA signal interference on the SU subcarrier. As an attacker attempts to fool SUs via mimicking the characteristics of the PU signal, the interference introduced by the $l$ th PUEA subcarrier to the SU $k$ that is using subcarrier $n$ can be calculated as follows:

$$
i_{k, n}^{E A}(l)=\left|h_{k, n}^{E S}\right|^{2} \int_{d_{n, l}-\Delta f / 2}^{d_{n, l}+\Delta f / 2} \Phi_{A}\left(e^{j \omega}\right) d \omega
$$

where $\Phi_{A}\left(e^{j \omega}\right)$ is the power spectrum density of the PUEA signal.

Then the interference introduced by the PUEA subcarriers that are incorrectly detected as PU signals in the set $O^{P}$, imposed to the SU $k$ that is using subcarrier $n$, can be written as

$$
j_{k, n}^{P}=\sum_{l \in O^{p}}\left(1-\alpha_{l}^{P}\right) i_{k, n}^{E A}(l)
$$

Similarly, the interference introduced by the PUEA subcarriers that are correctly detected as PUEA signals in the set $O^{A}$, imposed to the SU $k$ that is using subcarrier $n$, can be written as

$$
j_{k, n}^{A}=\sum_{l \in O^{A}} \alpha_{l}^{A} i_{k, n}^{E A}(l)
$$

Thus, the interference introduced by the PUEA signal to the SU $k$ that is using subcarrier $n$ can be written as

$$
j_{k, n}=j_{k, n}^{P}+j_{k, n}^{A}
$$

3.3. SU-PU Interference. This is the CRN interference on the $\mathrm{PN}$. Since SBS-SU is an OFDM signal, we assume an ideal Nyquist pulse shaping; the power density spectrum of the $n$th subcarrier in the SU subcarrier can be written as [22]

$$
\Phi_{n}^{\mathrm{SU}}(f)=p_{n} T_{s}\left(\frac{\sin \pi f T_{s}}{\pi f T_{s}}\right)^{2}
$$

where $p_{n}$ is the power loaded in the $n$th subcarrier and $T_{s}$ is the symbol duration.

The interference, introduced by the SU $k$ that is using subcarrier $n$, to the $l$ th PU subcarrier $\left(l \in O^{P} \cup O^{A}\right)$ can be written as follows:

$$
\begin{aligned}
i_{k, n}^{\mathrm{SU}}(l) & =C_{k, n}\left|h_{l}^{S P}\right|^{2} \int_{d_{n, l}-\Delta f / 2}^{d_{n, l}+\Delta f / 2} \Phi_{n}^{\mathrm{SU}}(f) d f \\
& =C_{k, n} p_{n}\left|h_{l}^{S P}\right|^{2} T_{s} \int_{d_{n, l}-\Delta f / 2}^{d_{n, l}+\Delta f / 2}\left(\frac{\sin \pi f T_{s}}{\pi f T_{s}}\right)^{2} d f \\
& =C_{k, n} p_{n}\left|h_{l}^{S P}\right|^{2} f\left(d_{n, l}\right),
\end{aligned}
$$

where

$$
f\left(d_{n, l}\right)=T_{s} \int_{d_{n, l}-\Delta f / 2}^{d_{n, l}+\Delta f / 2}\left(\frac{\sin \pi f T_{s}}{\pi f T_{s}}\right)^{2} d f .
$$

Using similar methods discussed in Section 3.1, the interference introduced by the SUs' signal to the $l$ th PU subcarrier $\left(l \in O^{P} \cup O^{A}\right)$ can be written as

$$
\begin{gathered}
I(l)=\sum_{k=1}^{K} \sum_{n=1}^{N} \alpha_{l}^{P} i_{k, n}^{\mathrm{SU}}(l), \quad l \in O^{P}, \\
I(l)=\sum_{k=1}^{K} \sum_{n=1}^{N}\left(1-\alpha_{l}^{A}\right) i_{k, n}^{\mathrm{SU}}(l), \quad l \in O^{A},
\end{gathered}
$$

respectively.

Hence, the $\mathrm{C} 1$ constraint in (6) can be written as

$$
\begin{gathered}
\operatorname{Pr}\left(\left|h_{l}^{S P}\right|^{2} \sum_{k=1}^{K} \sum_{n=1}^{N} C_{k, n} \alpha_{l}^{P} p_{n} f\left(d_{n, l}\right) \leq I_{\text {th }}^{(l)}\right) \geq a, \quad l \in O^{P}, \\
\operatorname{Pr}\left(\left|h_{l}^{S P}\right|^{2} \sum_{k=1}^{K} \sum_{n=1}^{N} C_{k, n}\left(1-\alpha_{l}^{A}\right) p_{n} f\left(d_{n, l}\right) \leq I_{\text {th }}^{(l)}\right) \geq a, \\
l \in O^{A} .
\end{gathered}
$$

Since the amplitude fading gain $\left|h_{l}^{S P}\right|$ is Rayleigh distributed with known parameter $\lambda_{l}$, the distribution of $\left|h_{l}^{S P}\right|^{2}$ is an exponential distribution. Hence, (20) can be written as

$$
\begin{aligned}
& 1-\exp \left\{-\frac{I_{\text {th }}^{(l)}}{2 \lambda_{l} \sum_{k=1}^{K} \sum_{n=1}^{N} C_{k, n} \alpha_{l}^{P} C_{k, n} p_{n} f\left(d_{n, l}\right)}\right\} \geq a, \\
& l \in O^{P} .
\end{aligned}
$$

Equation (22) can be rewritten as

$$
\sum_{k=1}^{K} \sum_{n=1}^{N} C_{k, n} \alpha_{l}^{P} C_{k, n} p_{n} f\left(d_{n, l}\right) \leq \frac{I_{\mathrm{th}}^{(l)}}{2 \lambda_{l}[-\ln (1-a)]}, \quad l \in O^{P} .
$$


Similarly, (21) can be written as

$$
\begin{array}{r}
\sum_{k=1}^{K} \sum_{n=1}^{N} C_{k, n}\left(1-\alpha_{l}^{A}\right) C_{k, n} p_{n} f\left(d_{n, l}\right) \leq \frac{I_{\mathrm{th}}^{(l)}}{2 \lambda_{l}[-\ln (1-a)]} \\
\quad l \in O^{A} .
\end{array}
$$

3.4. Subcarrier Allocation. The optimization problem defined by (6) is a mixed binary integer programming problem which is generally hard to solve. We decouple the optimization problem into two separate problems: a subcarrier allocation problem and a power allocation problem. In order to maximize the CRN system throughput, we allocate a particular subcarrier to a CR user that has the highest channel gain to interference and noise ratio (CINR).

Taking the background noise and interference from PU signal and PUEA signal into consideration, the CINR at SU $k$ that is using subcarrier $n$ can be expressed as

$$
\gamma_{k, n}=\frac{\left|h_{k, n}^{S S}\right|^{2}}{\sigma^{2}+i_{k, n}+j_{k, n}} .
$$

Theorem 1. Using the subcarrier allocation in (26), the optimization problem in (6) is decoupled into two separate problems, and the decoupling is optimal

$$
\begin{gathered}
C_{k, n}= \begin{cases}1, & k=k^{*} \\
0, & k \neq k^{*}\end{cases} \\
k^{*}=\arg \max _{k} \gamma_{k, n} .
\end{gathered}
$$

Proof. The proof is shown in Appendix A.

3.5. Power Allocation. After the subcarrier allocation, the optimization problem defined in (6) is turned into

$$
\begin{gathered}
\max _{p_{k, n}} \quad \Delta f \sum_{k=1}^{K} \sum_{n=\Omega_{k}} \log _{2}\left(1+\frac{p_{k, n}\left|h_{k, n}^{S S}\right|}{\sigma^{2}+i_{k, n}+j_{k, n}}\right) \\
\text { s.t. } \quad \mathrm{C} 1: \sum_{k=1}^{K} \sum_{n=\Omega_{k}} \alpha_{l}^{P} C_{k, n} p_{n} f\left(d_{n, l}\right) \\
\leq \frac{I_{\text {th }}^{(l)}}{2 \lambda_{l}[-\ln (1-a)]}, \quad l \in O^{P} \\
\text { C2: } \sum_{k=1}^{K} \sum_{n=\Omega_{k}} C_{k, n}\left(1-\alpha_{l}^{A}\right) C_{k, n} p_{n} f\left(d_{n, l}\right) \\
\leq \frac{I_{\text {th }}^{(l)}}{2 \lambda_{l}[-\ln (1-a)]}, \quad l \in O^{A} \\
\text { C3: } p_{k, n} \geq 0, \quad \forall k \in \mathscr{K}, \quad \forall n \in \mathscr{N} \\
\text { C4: } \sum_{k=1}^{K} \sum_{n=\Omega_{k}} p_{k, n} \leq P_{T},
\end{gathered}
$$

where $\Omega_{k}$ denotes the set of subcarriers allocated to the $k$ th SU according to (26).

Theorem 2. The optimal power allocated to nth subcarrier that the kth $S U$ is using can be written as

$$
\begin{aligned}
p_{k, n}^{*}= & {\left[1 \times\left(\operatorname { l n } 2 \left[\theta+\sum_{l \in O^{P}} \beta_{l} \alpha_{l}^{P} f\left(d_{n, l}\right)\right.\right.\right.} \\
& \left.\left.+\sum_{l \in O^{A}} \rho_{l}\left(1-\alpha_{l}^{A}\right) f\left(d_{n, l}\right)\right]\right)^{-1} \\
& \left.-\frac{\sigma^{2}+i_{k, n}+j_{k, n}}{\left|h_{k, n}^{S S}\right|}\right]^{+} \quad \forall k \in \mathscr{K}, n \in \Omega_{k},
\end{aligned}
$$

where $[x]^{+}=\max (0, x), \theta, \beta_{l}\left(l \in O^{P}\right)$, and $\rho_{l}\left(l \in O^{A}\right)$ are Lagrange constants.

The proof of Theorem 2 and the method of calculating the optimal power allocation $p_{k, n}^{*}$ and the Lagrange multipliers are given in Appendix B.

3.6. Proposed Rope-Alone. The whole process of the proposed Rope-Alone can be summarized in Algorithm 1.

\section{Simulations and Discussions}

4.1. Simulation Settings. We give simulation results to evaluate the performance of the proposed algorithm in this section. The total available bandwidth $(W)$, which is equally divided into 16 OFDM subcarriers, is assumed to be equal to $8 \mathrm{MHz}$. So the bandwidth $\Delta f$ is $0.5 \mathrm{MHz}$ for each subcarrier. The values of $T_{s}$ and $\sigma^{2}$ have been taken to be $4 \mu \mathrm{s}$ and $10^{-7}$ Watt, respectively. We assumed that there are $K=4$ SUs. The channel fading gains $h_{l}^{S P}, h_{k, n}^{S S}$, and $h_{k, n}^{P S}$ are assumed to be Rayleigh distributed with mean values of $-46 \mathrm{~dB},-40 \mathrm{~dB}$, and $-54 \mathrm{~dB}$, respectively. In addition, the channel fading gain $h_{l}^{E P}$ is assumed to be Rayleigh distributed with mean values of $-46 \mathrm{~dB}$. The transmit power of the PU is assumed to be $0.1 \mathrm{~W}$. The probability $a$, with which the interference introduced to the PU $l$ th subcarrier remains below the threshold $I_{\mathrm{th}}^{l}$, is equal to 0.95 .

We also assume that, after the robust spectrum sensing, all of these subcarriers will be divided into three parts: $V=[1,2,6,7,8,12,13,16], O^{P}=[3,4,5,9,10]$, and $O^{A}=$ $[11,14,15]$. To analyze the performance of the proposed Rope-Alone, we give the detection performance of the robust spectrum sensing being used. Given $P_{f}^{P}=0.06$, the $P_{d}^{P}$ is equal to $[0.99,0.98,0.97,0.98,0.96]$ over subcarriers in the set $O^{P}$. In addition, the $P_{d}^{A}$ is equal to $[0.97,0.98,0.96]$ over subcarriers in the set $O^{A}$. The probability of PU activity in each subcarrier follows normal distribution with mean 0.7 and variance 0.01 .

4.2. Impact of Power Budget. For a given value of interference threshold $I_{\text {th }}^{l}=5 \times 10^{-9}$ Watt for PU subcarrier $l, l \in O^{P} \cup O^{A}$, 


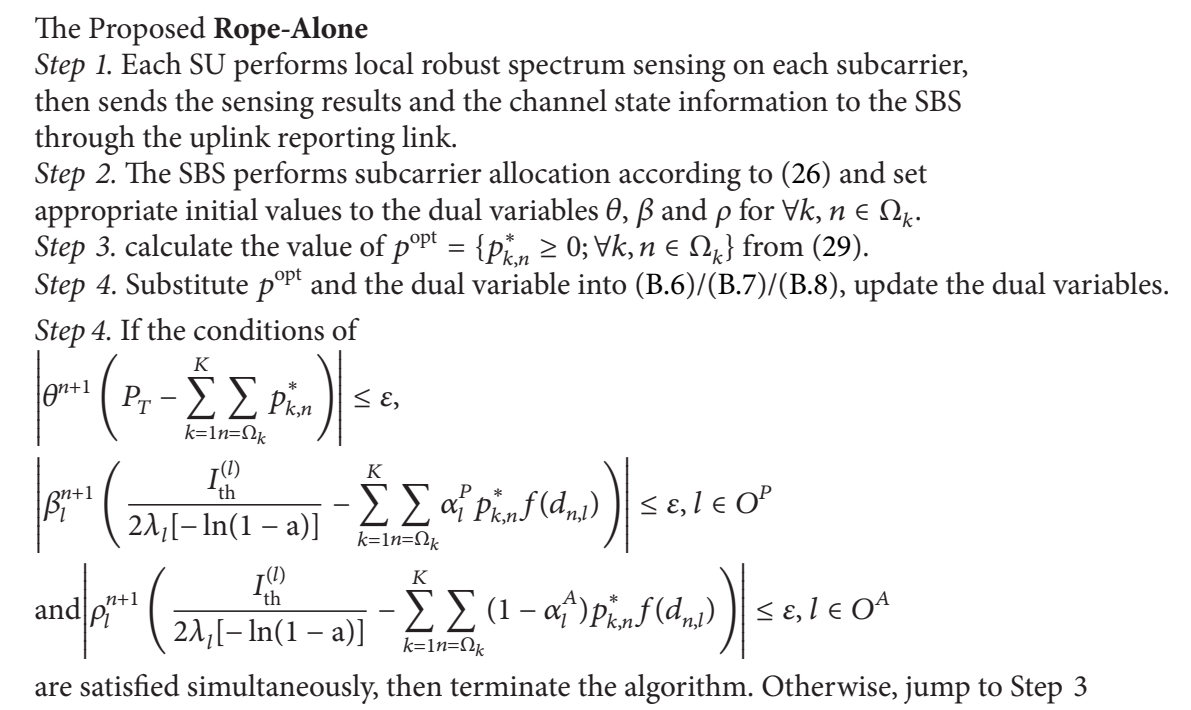

Algorithm 1: Robust cooperative spectrum sensing-assisted multiuser resource allocation scheme.

we study the performance of the proposed Rope-Alone and conventional scheme [12] in subcarrier-and-power allocation in Figure 3. The conventional scheme in subcarrier-andpower allocation makes no distinction between the subcarriers in set $O^{P}$ and the subcarriers in set $O^{A}$ and extends the same protection to all subcarrier $l, l \in O^{P} \cup O^{A}$. In Figure 3, we have plotted the maximum system throughput of the CRN versus the total power budget for Rope-Alone and conventional scheme with the HOUSAM. In this figure, we have also plotted the maximum system throughput of the CRN for Rope-Alone and conventional scheme with the OSAM. It can be observed that both schemes with the HOUSAM achieve higher transmission rate compared with OSAM. Furthermore, regardless of the spectrum access mechanism (OSAM or HOUSAM) being used, the achievable maximum system throughput for the Rope-Alone is greater than that of the conventional scheme.

In Figure 3, we also observed that, for the conventional scheme when the power budget exceeds $180 \mathrm{~mW}$, the maximum system throughout becomes roughly constant, while for Rope-Alone this power budget threshold is approximately $370 \mathrm{~mW}$. This is due to the fact that, when the power budget exceeds a certain threshold, these schemes will operate in the interference-limited scenario; that is, interference constraints $\mathrm{C} 1$ in (28) become the boundary constraint, and the total power loaded on all subcarriers became a constant and does not change with power budget increases. To gain a clearer vision of how much power can be loaded into different subcarriers under diffident power budget, Figures 4 and 5 provide the power loading profile for Rope-Alone and conventional scheme under various spectrum access mechanisms with the power budget of $160 \mathrm{~mW}$ and $300 \mathrm{~mW}$, respectively. In Figures 4 and 5, we also have fixed the values of $I_{\text {th }}^{l}=5$ $\times 10^{-9}$. From Figure 5, it can be observed that the power loaded in some subcarriers with Rope-Alone is much higher

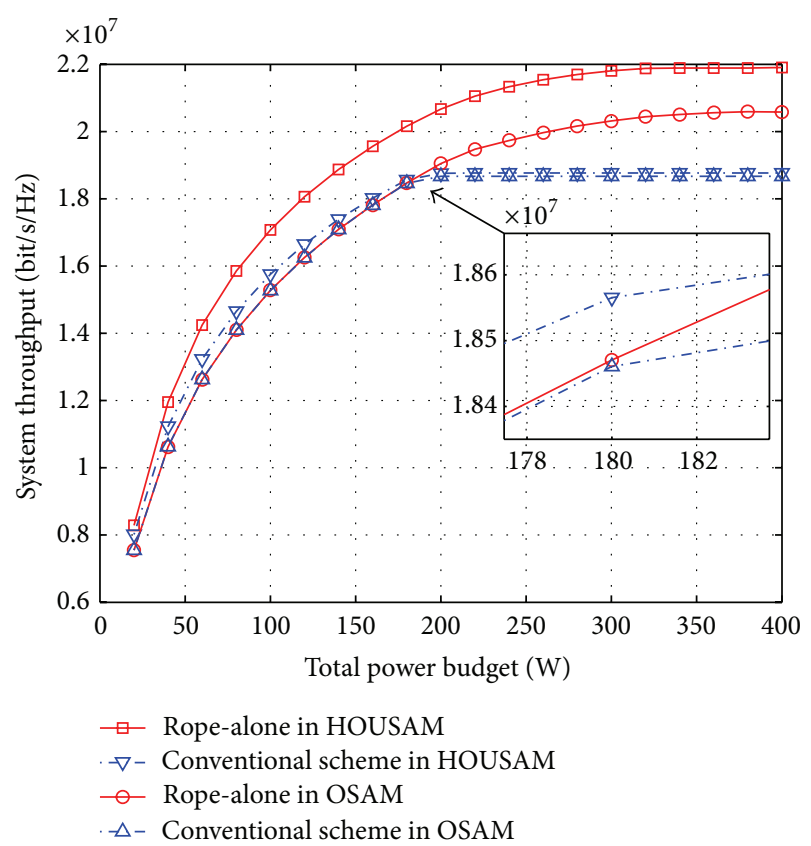

FIGURE 3: System throughput versus total power budget for various schemes.

than that with conventional scheme as expected. We find that, owning to the assist of robust cooperative spectrum sensing, the proposed Rope-Alone could exploit spectrum opportunity more effectively; that is, making full use of the sensing information to some extent means better system performance.

4.3. Impact on Energy Efficiency. We define an energy efficiency metric, $\psi$, as the ratio between the system throughput 


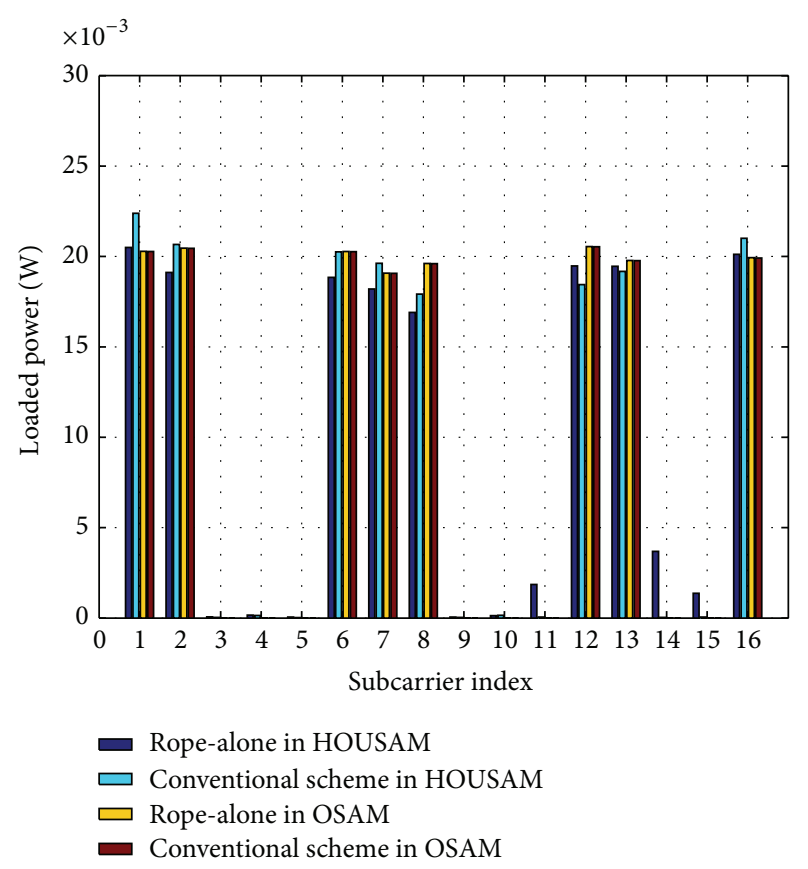

FIgUre 4: Power profile for various schemes (power budget = $160 \mathrm{~mW})$.

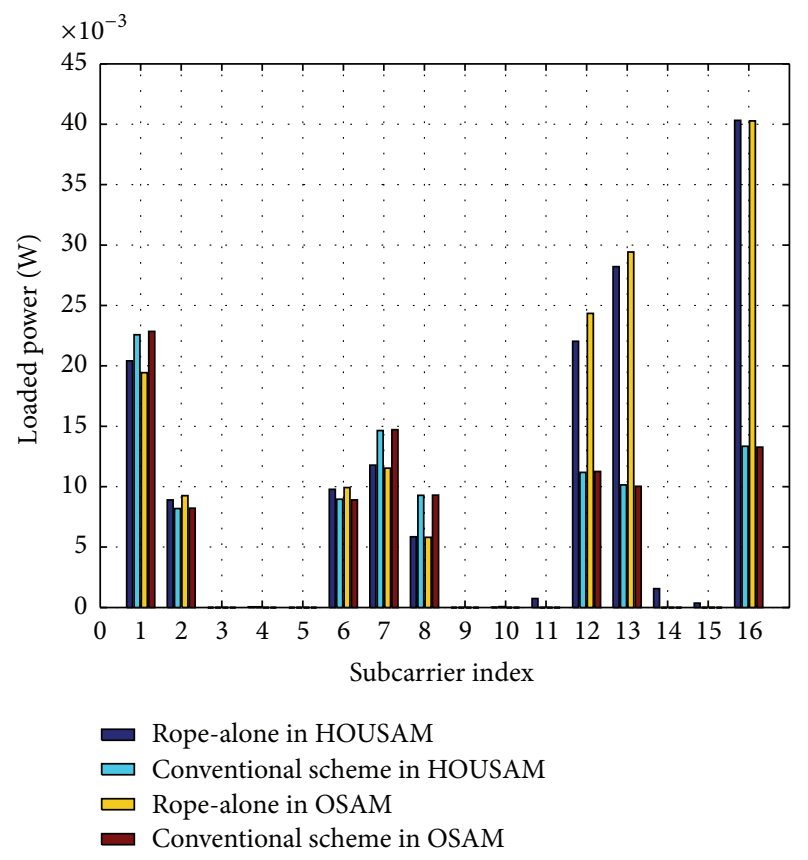

FIGURE 5: Power profile for various schemes (power budget = $300 \mathrm{~mW})$.

and the total achievable transmission power. To see the performance of energy efficiency of the Rope-Alone and the conventional scheme allocation schemes, we further plotted the energy efficiency $\psi$ versus the total power budget for both schemes with HOUSAM and OSAM. In Figure 6, we have fixed the values of $I_{\text {th }}^{l}=5 \times 10^{-9}$ for all $l$. From

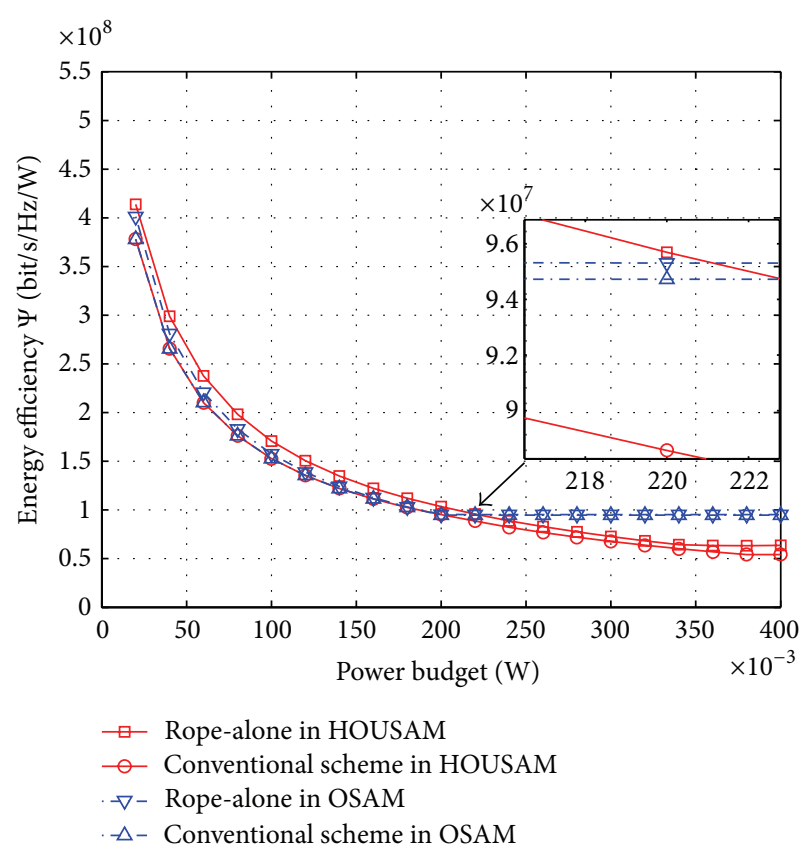

Figure 6: Energy efficiency $\psi$ versus total power budget.

Figure 6, we notice that the energy efficiency of Rope-Alone is higher than that of conventional scheme, regardless of the spectrum access mechanism (OSAM or HOUSAM) being used. We also find when the power budget is over $220 \mathrm{~mW}$, the energy efficiency of the HOUSAM based scheme is smaller than that of the OSAM based scheme. However, in Figure 3, we find that the achievable system throughput with the HOUSAM based scheme is higher than that of the OSAM based scheme when the power budget is over $220 \mathrm{~mW}$. This fact demonstrates that the performance improvement of the HOUSAM based scheme comes at the expense of energy efficiency.

4.4. Impact of Interference Threshold. Figure 7 shows the impact of interference threshold $I_{\text {th }}^{l}$ on the system throughput in different schemes. For this plotting, we have used power budget $P_{T}=100 \mathrm{~mW}$. We can see that, for all schemes, the system throughput increases almost linearly as the interference threshold $I_{\text {th }}^{l}$ increases up to a certain value of interference threshold and then it becomes roughly constant. The reason behind this is that, for the given power budget, with the interference threshold increasing, the interference constraint is no longer a boundary constraint, but the power budget becomes the boundary constraint which restricts the improvement of the system throughput. Moreover, we can see that Rope-Alone outperforms the conventional scheme under all spectrum access mechanisms.

4.5. Impact of Secondary Users. The system throughput versus number of secondary users for various schemes is shown in Figure 8. In this figure, we have fixed the values of $I_{\text {th }}^{l}=5$ $\times 10^{-9}$ and the total power budget $P_{T}$ to be $250 \mathrm{~mW}$. It is shown that the system throughput increases with the number 


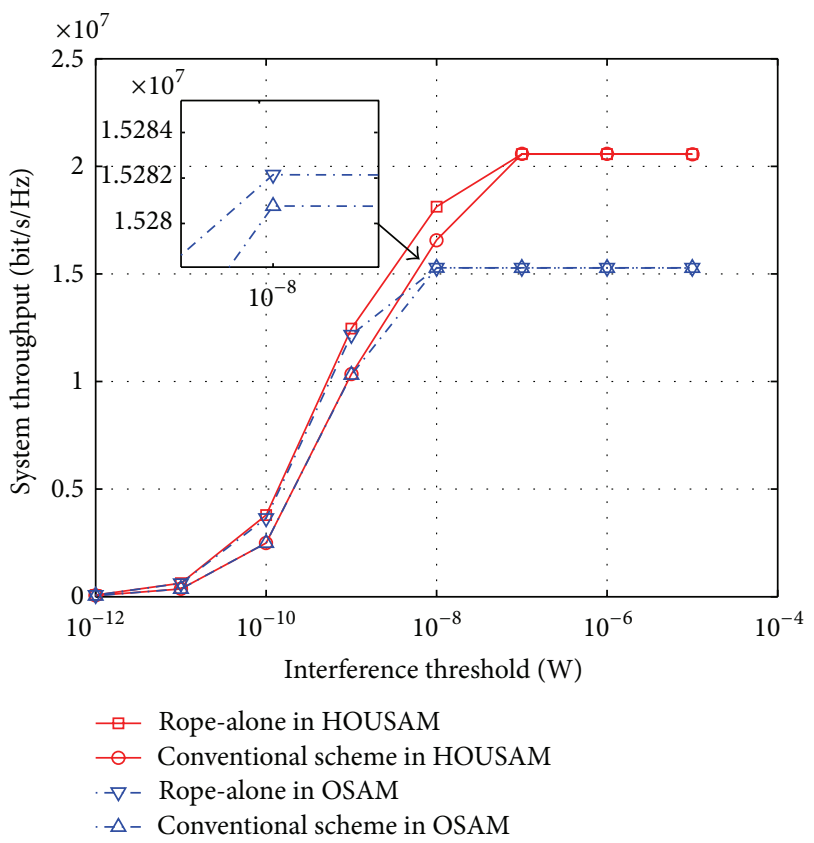

FIGURE 7: System throughput versus interference threshold for various schemes.

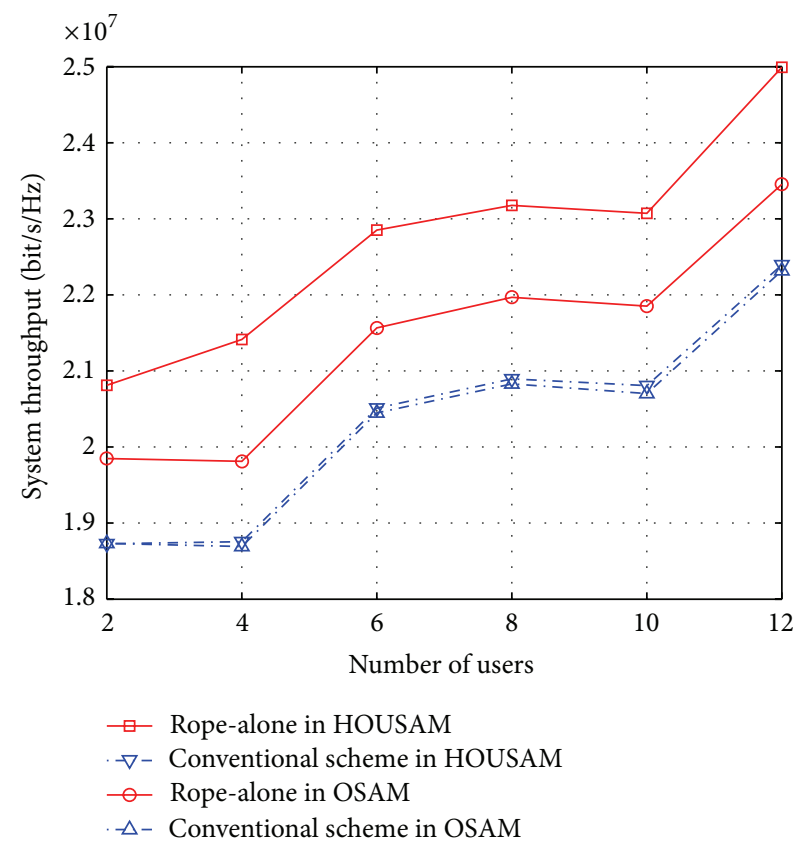

FIGURE 8: System throughput versus number of secondary users for various schemes.

of SUs. This is mainly because the more the number of SUs in CRN, the more likely for the CRN to have high quality channel. In addition, the Rope-Alone performs better than the conventional scheme regardless of the number of SUs.

4.6. Impact of the Accuracy of Detecting the PUEA Signal. We evaluate the impact on the system throughput when varying

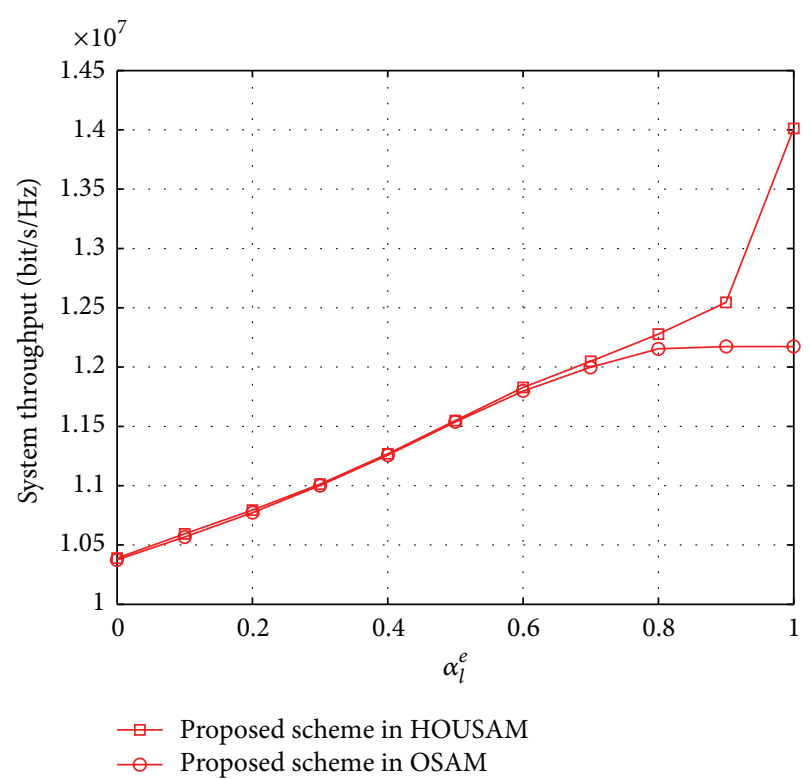

FIGURE 9: System throughput versus the accuracy of detecting the PUEA signal.

the accuracy of detecting the PUEA signal for the robust cooperative spectrum sensing. In Figure 9, we have fixed the values of $I_{\text {th }}^{l}$ to be $5 \times 10^{-9}$ Watt and total power budget to $100 \mathrm{~mW}$. We have plotted $\alpha_{l}^{A}$ (the accuracy of detecting the PUEA signal) versus system throughput for various schemes under consideration. The value $\alpha_{l}^{A}$ is determined by the performance of robust cooperative spectrum sensing to differentiate PUEA signal from PU signal. Higher $\alpha_{l}^{A}$ means less ambiguity between PUEA signals from PU signals. As can be observed from Figure 9, the system throughput increases as $\alpha_{l}^{A}$ increases as expected. When $\alpha_{l}^{A}$ is close to 1 , the improvement in system throughput with HOUSAM is much higher than that with OSAM.

\section{Conclusion}

How to maximize the system throughput of OFDMA-based CRN while keeping the interference introduced to the PU below certain thresholds in the present of a PUEA is a challenging issue. To address this problem, we studied the joint design of robust spectrum sensing and resource allocation in OFDMA-based CRN and formulated a joint design scheme as a convex optimization problem. The Lagrange duality theory has been introduced to solve this problem. To further exploit the underutilized spectrum bands, we also have evaluated the performance of the proposed scheme in the hybrid overlay/underlay spectrum access mechanism. Simulation results have confirmed the superiority of our proposed RopeAlone compared to conventional resource allocation scheme under various spectrum access mechanisms. These results also have shown that the Rope-Alone achieves higher energy efficiency than conventional scheme. The proposed RopeAlone is mainly for stationary SUs and may be applicable to low speeds; an interesting challenge ahead is to design 
a robust resource allocation scheme applicable to OFDMAbased medium/high speed mobile CRN. Since the high mobility of the SU leads to Doppler frequency spread, which will give rise to intercarrier interference. Such intercarrier interference severely affects the performance of OFDMAbased CRN.

\section{Appendices}

\section{A. Proof of Theorem 1}

Let us prove Theorem 1 with reduction to absurdity. Assume an optimal subcarrier allocation exists and $\mathrm{SU} k_{1}$ in the $n$th subcarrier with the highest CINR $\gamma_{k_{1}, n}$. However, the $n$th subcarrier is assigned to SU $k_{2}$ with the CINR $\gamma_{k_{2}, n}$ smaller than $\gamma_{k_{1}, n}$. Let us assume that the optimal power $p_{k_{2}, n}$ is allocated to SU $k_{2}$ in the $n$th subcarrier which satisfies constraints C1-C5 in (6). If we allocate $n$th subcarrier with the power $p_{k_{2}, n}$ to SU $k_{1}$, the constraints $\mathrm{C} 1-\mathrm{C} 5$ will still be satisfied. But CRN system throughput in (6) will be higher as $\gamma_{k_{1}, n} p_{k_{2}, n}>\gamma_{k_{2}, n} p_{k_{2}, n}$. Therefore, assigning the $n$th subcarrier to $\mathrm{SU} k_{2}$ is not optimal. Hence, Theorem 1 is proven by reduction to absurdity.

\section{B. Proof of Theorem 2}

To make the optimization problem in (28) tractable, we solve it by considering its Lagrange dual problem. It is known that if the optimization problem is convex, the duality gap is zero. As a result, the optimal solution obtained by the duality problem is the global optimal solution of the primary problem [23]. The optimization problem specified in (28) is convex with respect to the optimization variable $p_{k, n}$.

Firstly, by introducing nonnegative dual variables $\theta, \boldsymbol{\beta}=$ $\left[\beta_{1}, \beta_{2}, \ldots, \beta_{\left|O^{P}\right|}\right]$ and $\boldsymbol{\rho}=\left[\rho_{1}, \rho_{2}, \ldots, \rho_{\left|O^{A}\right|}\right]$, the Lagrange function is given by

$$
\begin{aligned}
L(\mathbf{p}, \boldsymbol{\beta}, \boldsymbol{\rho}, \theta)= & \sum_{k=1}^{K} \sum_{n=\Omega_{k}} \log _{2}\left(1+\frac{p_{k, n}\left|h_{k, n}^{S S}\right|}{\sigma^{2}+i_{k, n}+j_{k, n}}\right) \\
& +\sum_{l \in O^{P}} \beta_{l}\left(\frac{I_{\mathrm{th}}^{(l)}}{2 \lambda_{l}[-\ln (1-a)]}\right. \\
& +\sum_{l \in \mathrm{O}^{A}} \rho_{l}\left(\frac{\left.-\sum_{k=1}^{K} \sum_{n=\Omega_{k}} \alpha_{l}^{P} p_{k, n} f\left(d_{n, l}\right)\right)}{2 \lambda_{l}[-\ln (1-a)]}\right. \\
& +\theta\left(P_{T}^{(l)}-\sum_{k=1}^{K} \sum_{n=\Omega_{k}} p_{k, n}\right),
\end{aligned}
$$

where $\mathbf{p}=\left\{p_{k, n} \geq 0 ; \forall k, n \in \Omega_{k}\right\},\left|O^{P}\right|$ is the cardinality of the set $O^{P}$ and $\left|O^{A}\right|$ is the cardinality of the set $O^{A}$.

Secondly, Lagrange dual function can be obtained by

$$
D(\boldsymbol{\beta}, \boldsymbol{\rho}, \theta)=\max _{\mathbf{p}} L(\mathbf{p}, \boldsymbol{\beta}, \boldsymbol{\rho}, \theta)
$$

and the dual problem can be written as

$$
d^{*}=\min _{\theta \geq 0, \beta \geq 0, \rho \geq 0} D(\boldsymbol{\beta}, \boldsymbol{\rho}, \theta) \text {. }
$$

The dual problem in (B.3) can be decomposed into the following two sequentially iterative subproblems.

Subproblem 1 (Power Allocation). Given the dual variable $\theta$, $\boldsymbol{\beta}$ and $\boldsymbol{\rho}$, for $\forall k, n \in \Omega_{k}$, the objective function (B.1) is maximized when the derivative with respect to $p_{k, n}$ is zero; the optimized power allocation for each subcarrier can be written as

$$
\begin{gathered}
p_{k, n}^{*}=\left[1 \times\left(\operatorname { l n } 2 \left[\theta+\sum_{l \in O^{P}} \beta_{l} \alpha_{l}^{P} f\left(d_{n, l}\right)\right.\right.\right. \\
\left.\left.+\sum_{l \in O^{A}} \rho_{l}\left(1-\alpha_{l}^{A}\right) f\left(d_{n, l}\right)\right]\right)^{-1} \\
\left.-\frac{\sigma^{2}+i_{k, n}+j_{k, n}}{\left|h_{k, n}^{S S}\right|}\right]^{+}, \quad \forall k, n \in \Omega_{k} .
\end{gathered}
$$

Subproblem 2 (Dual Variable Update). The optimal dual variable can be obtained by solving the dual problem:

$$
\begin{aligned}
& \left(\boldsymbol{\beta}^{\mathrm{opt}}, \boldsymbol{\rho}^{\mathrm{opt}}, \theta^{\mathrm{opt}}\right) \\
& \quad=\arg \max _{\theta \geq 0, \beta \geq 0, \rho \geq 0} \min \left[L\left(\mathbf{p}^{\mathrm{opt}}, \boldsymbol{\beta}, \boldsymbol{\rho}, \theta\right)\right],
\end{aligned}
$$

where $\mathbf{p}^{\text {opt }}=\left\{p_{k, n}^{*} \geq 0 ; \forall k, n \in \Omega_{k}\right\}$. The Lagrange multipliers $\boldsymbol{\beta}^{\text {opt }}, \boldsymbol{\rho}^{\mathrm{opt}}$, and $\theta^{\mathrm{opt}}$ in (B.5) can be calculated using the interior point method; here we can use a subgradient update method (a generalization of gradient) [23]:

$$
\theta^{n+1}=\left[\theta^{n}-\Delta_{\theta}^{n}\left(P_{T}-\sum_{k=1}^{K} \sum_{n=\Omega_{k}} p_{k, n}^{*}\right)\right]^{+},
$$




$$
\begin{gathered}
\beta_{l}^{n+1}=\left[\beta_{l}^{n}-\Delta_{\beta}^{n}\left(\frac{I_{\mathrm{th}}^{(l)}}{2 \lambda_{l}[-\ln (1-a)]}\right.\right. \\
\left.\left.-\sum_{k=1}^{K} \sum_{n=\Omega_{k}} \alpha_{l}^{P} p_{k, n}^{*} f\left(d_{n, l}\right)\right)\right]^{+}, \\
\rho_{l}^{n+1}=\left[\rho_{l}^{n}-\Delta_{\rho}^{n}\left(\frac{I_{\mathrm{th}}^{(l)}}{2 \lambda_{l}[-\ln (1-a)]} \quad l \in O^{P},\right.\right. \\
\left.\left.-\sum_{k=1}^{K} \sum_{n=\Omega_{k}}\left(1-\alpha_{l}^{A}\right) p_{k, n}^{*} f\left(d_{n, l}\right)\right)\right]^{+},
\end{gathered}
$$

where $n$ is the iteration number and $\Delta$ is the iteration step size.

According to the theoretical results in [24], the above dual variable updating method is guaranteed to converge to the optimal solution, as long as the iteration step chosen is sufficiently small.

\section{Abbreviations}

AT: $\quad$ Attacker's transmitter

CINR: Channel gain to interference and noise

CR: $\quad$ ratio $\quad$ Cognitive radio

CRN: Cognitive radio networks

HOUSAM: Hybrid overlay/underlay spectrum access mechanism

OFDM: Orthogonal frequency division multiplexing

OFDMA: Orthogonal frequency division multiple access

OSAM: $\quad$ Overlay spectrum access mechanism

PN: $\quad$ Primary user network

PR: $\quad$ Primary user's receiver

PT: $\quad$ Primary user's transmitter

PU: $\quad$ Primary user

PUEA: Primary user emulation attack

Rope-Alone: Robust cooperative spectrum sensing-assisted multiuser resource allocation scheme

SBS: $\quad$ Secondary base station

SR: $\quad$ Secondary user's receiver

SU: $\quad$ Secondary user

TDMA: Time division multiple access

USAM: Underlay spectrum access mechanism.

\section{Conflict of Interests}

The authors declare that there is no conflict of interests regarding the publishing of this paper.

\section{Acknowledgment}

The authors would like to thank the National High-Tech Research and Development Program of China (863 Program) under Grant 2012AA01A508 for the project support.

\section{References}

[1] J. Mitola and G. Q. Maguire Jr., "Cognitive radio: making software radios more personal," IEEE Personal Communications, vol. 6, no. 4, pp. 13-18, 1999.

[2] K. Muthumeenakshi and S. Radha, "Distributed cognitive radio spectrum access with imperfect sensing using CTMC," International Journal of Distributed Sensor Networks, vol. 2013, Article ID 257801, 11 pages, 2013.

[3] J. Zou, H. Xiong, D. Wang, and C. W. Chen, "Optimal power allocation for hybrid overlay/underlay spectrum sharing in multiband cognitive radio networks," IEEE Transactions on Vehicular Technology, vol. 62, no. 4, pp. 1827-1837, 2013.

[4] H. Mahmoud, T. Yücek, and H. Arslan, "OFDM for cognitive radio: merits and challenges," IEEE Wireless Communications, vol. 16, no. 2, pp. 6-15, 2009.

[5] J. Huang, V. G. Subramanian, R. Agrawal, and R. A. Berry, "Downlink scheduling and resource allocation for OFDM systems," IEEE Transactions on Wireless Communications, vol. 8, no. 1, pp. 288-296, 2009.

[6] K. Kim, Y. Han, and S. Kim, "Joint subcarrier and power allocation in uplink OFDMA systems," IEEE Communications Letters, vol. 9, no. 6, pp. 526-528, 2005.

[7] E. Yaacoub and Z. Dawy, "A survey on uplink resource allocation in OFDMA wireless networks," IEEE Communications Surveys and Tutorials, vol. 14, no. 2, pp. 322-337, 2012.

[8] I. F. Akyildiz, B. F. Lo, and R. Balakrishnan, "Cooperative spectrum sensing in cognitive radio networks: a survey," Physical Communication, vol. 4, no. 1, pp. 40-62, 2011.

[9] F. Bao, H. Chen, and L. Xie, "Analysis of primary user emulation attack with motional secondary users in cognitive radio networks," in Proceeding of the 23rd IEEE International Symposium on Personal, Indoor and Mobile Radio Communications (PIMRC '12), pp. 956-961, Sydney, Australia, September 2012.

[10] C. Chen, H. Cheng, and Y. Yao, "Cooperative spectrum sensing in cognitive radio networks in the presence of the primary user emulation attack," IEEE Transactions on Wireless Communications, vol. 10, no. 7, pp. 2135-2141, 2011.

[11] M. Haghighat and S. M. Sajad Sadough, "Cooperative spectrum sensing for cognitive radio networks in the presence of smart malicious users," AEU-International Journal of Electronics and Communications, vol. 68, no. 6, pp. 520-527, 2014.

[12] G. Bansal, M. J. Hossain, V. K. Bhargava, and T. Le-Ngoc, "Subcarrier and power allocation for OFDMA-based cognitive radio systems with joint overlay and underlay spectrum access mechanism," IEEE Transactions on Vehicular Technology, vol. 62, no. 3, pp. 1111-1122, 2013.

[13] G. Ding, Q. Wu, and J. Wang, "Sensing confidence levelbased joint spectrum and power allocation in cognitive radio networks," Wireless Personal Communications, vol. 72, article 826, 2013.

[14] S. Parsaeefard and A. R. Sharafat, "Robust distributed power control in cognitive radio networks," IEEE Transactions on Mobile Computing, vol. 12, no. 4, pp. 609-620, 2013. 
[15] M. Haghighat, H. Fathi, and S. M. Sadough, "Robust resource allocation for OFDM-based cognitive radio in the presence of primary user emulation attack," Radioengineering, vol. 21, no. 4, pp. 1085-1091, 2012.

[16] K. Bian and J.-M. J. Park, "Security vulnerabilities in IEEE 802.22," in Proceedings of the 4th Annual International Conference on Wireless Internet, p. 9, 2008.

[17] A. G. Fragkiadakis, E. Z. Tragos, and I. G. Askoxylakis, "A survey on security threats and detection techniques in cognitive radio networks," IEEE Communications Surveys and Tutorials, vol. 15, no. 1, pp. 428-445, 2013.

[18] B. F. Lo, "A survey of common control channel design in cognitive radio networks," Physical Communication, vol. 4, no. 1, pp. 26-39, 2011.

[19] J. He, C. Xu, and L. Li, "Adaptive subcarrier bandwidth and power in OFDM-based cognitive radio systems for high mobility applications," International Journal of Communication Systems, 2012.

[20] S. S. Das, E. de Carvalho, and R. Prasad, "Performance analysis of OFDM systems with adaptive sub carrier bandwidth," IEEE Transactions on Wireless Communications, vol. 7, no. 4, pp. 1117$1122,2008$.

[21] T. M. Cover and J. A. Thomas, Elements of Information Theory, John Wiley \& Sons, New York, NY, USA, Second edition, 2006.

[22] T. Weiss, J. Hillenbrand, A. Krohn, and F. K. Jondral, "Mutual interference in OFDM-based spectrum pooling systems," in Proceedings of the IEEE 59th Vehicular Technology Conference (VTC '04), vol. 4, pp. 1873-1877, May 2004.

[23] S. P. Boyd and L. Vandenberghe, Convex Optimization, Cambridge University Press, Cambridge, UK, 2004.

[24] W. Yu and R. Lui, "Dual methods for nonconvex spectrum optimization of multicarrier systems," IEEE Transactions on Communications, vol. 54, no. 7, pp. 1310-1322, 2006. 


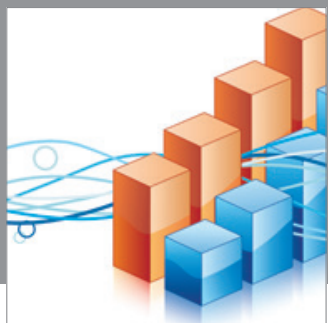

Advances in

Operations Research

mansans

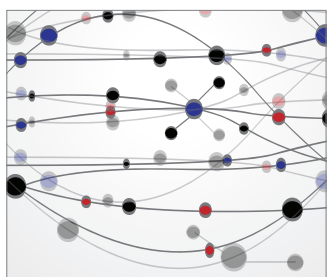

The Scientific World Journal
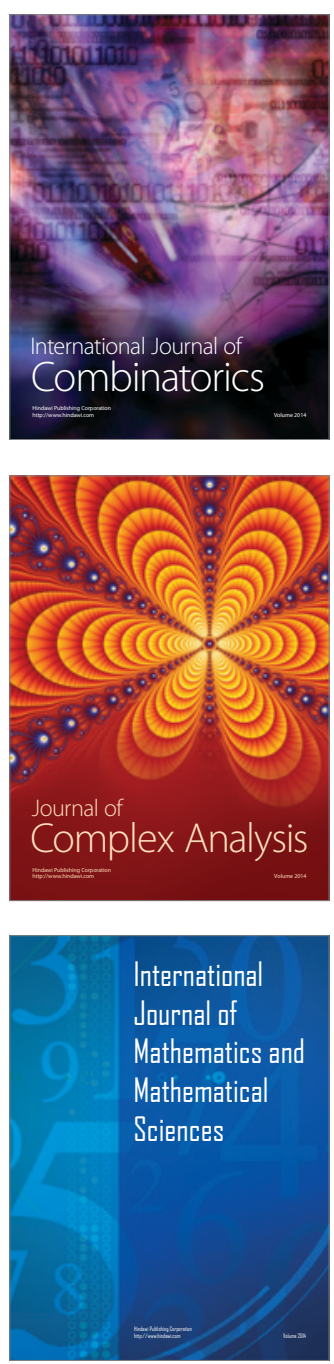
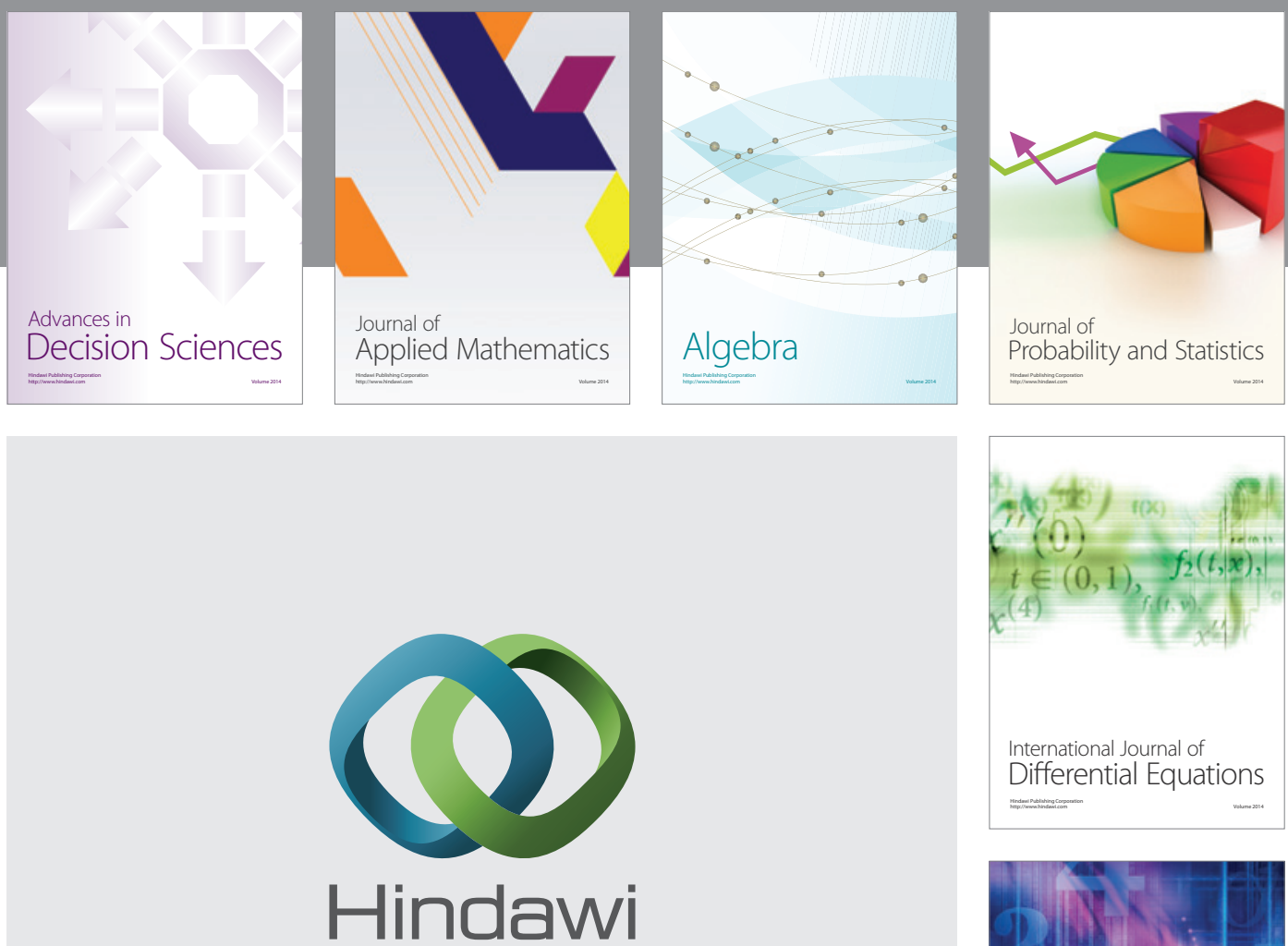

Submit your manuscripts at http://www.hindawi.com
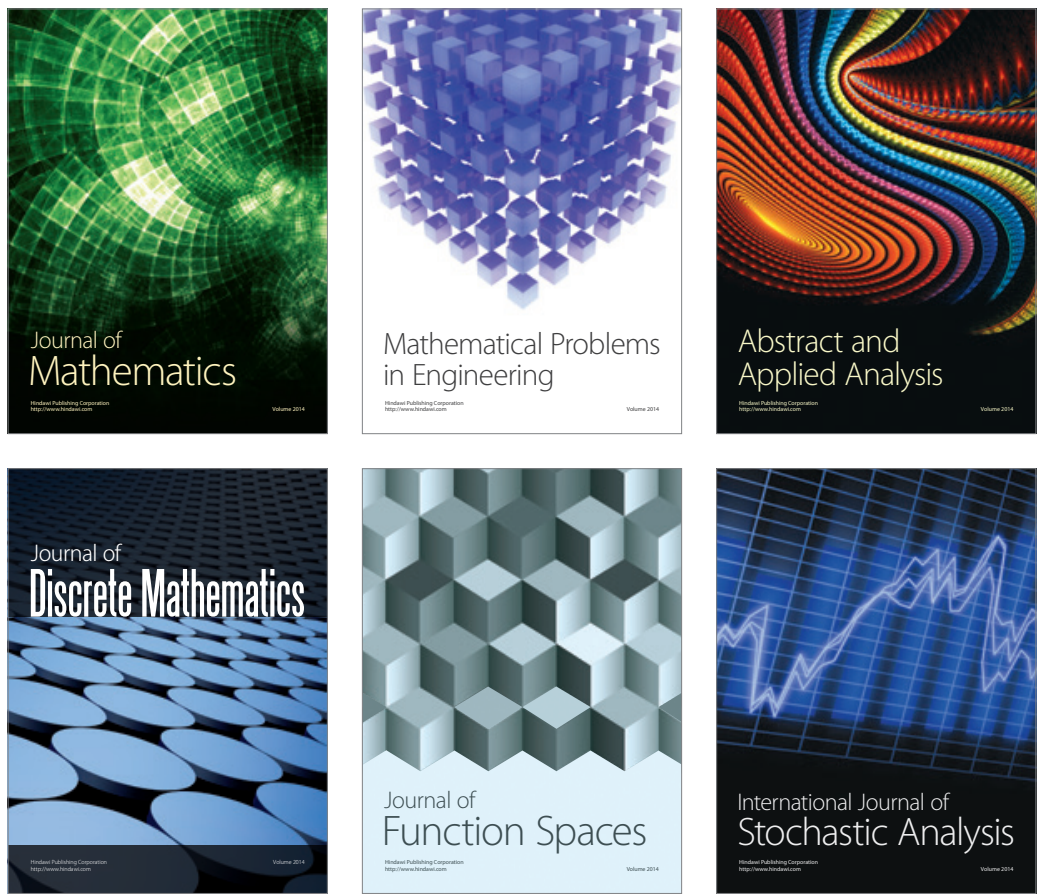

Journal of

Function Spaces

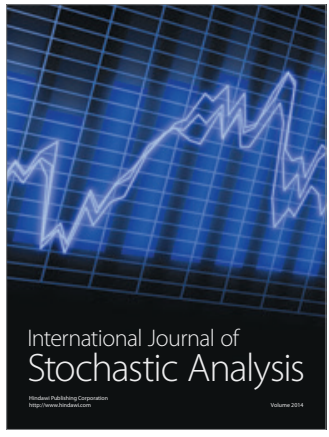

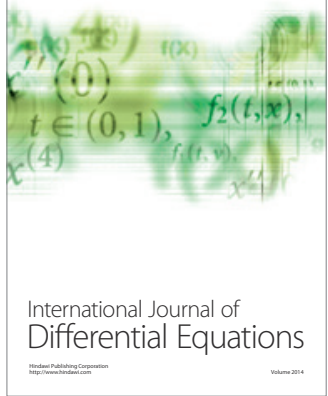
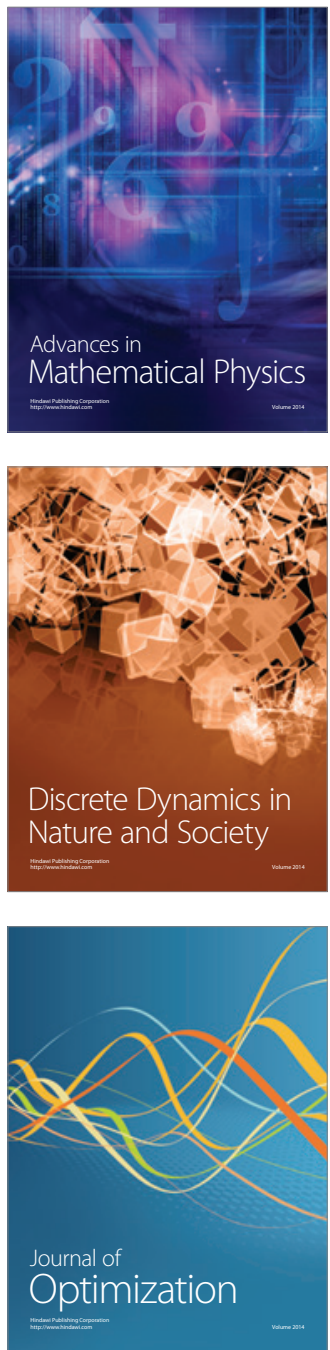